\title{
ARTICLE
}

\section{Evaluation of dose level in a laser-driven ion accelerator using PHITS code}

\author{
Hironao Sakaki $^{{ }^{*}}$, Yuji Fukuda ${ }^{\mathrm{a}}$, Mamiko Nishiuchi ${ }^{\mathrm{a}}$, Satoshi Jinno ${ }^{\mathrm{a}}$, Masato Kanasaki ${ }^{\mathrm{ab}}$, Akifumi Yogo ${ }^{\mathrm{a}}$, \\ Kiminori Kondo ${ }^{\mathrm{a}}$, Fumihiro Saito ${ }^{\mathrm{a}}$, Tomoyo Fukami ${ }^{\mathrm{a}}$, Masayuki Ueno ${ }^{\mathrm{a}}$ and Koji Niita ${ }^{\mathrm{c}}$ \\ ${ }^{a}$ Japan Atomic Energy Agency, 8-1-7 Umemi-dai, Kizugawa, Kyoto 619-0215, Japan; ${ }^{b}$ Graduate School of Maritime Sciences, Kobe \\ University, 5-1-1, Fukaeminami, Higashinada, Kobe 658-0022, Japan; ${ }^{c}$ Research Organization for Information Science and \\ Technology, 2-4, Shirakata, Tokai, Ibaraki 319-1106, Japan
}

The laser-driven particle accelerator has become attractive in view of recent progress in laser-handling techniques and the development of various target materials. To develop a laser-driven accelerator, it is necessary to establish a benchmark for the difference between the simulated and measured radiation shielding level. The Monte Carlo particle and heavy ion transport code system (PHITS) was used to establish the benchmark dose for a laser-driven cluster-target-type accelerator. The result was in good agreement with the measurement data.

Keywords: laser-driven accelerator; Monte Carlo; PHITS; dose distributions

\section{Introduction}

With the development of new laser systems and handling techniques, a focused laser intensity of over $10^{22} \mathrm{~W} / \mathrm{cm}^{2}$ [1] (ultrashort pulse high-intensity lasers) has become possible with the use of a table-sized petawatt laser system. This ultrashort pulse laser is mainly applied to the study of laser-plasma interactions that generate high-intensity ( 100 GV/m) electric fields [2-4]. To study this electric field, we aimed to realize a small laser-driven ion accelerator that exceeds the energy of a conventional accelerator such as a synchrotron or cyclotron.
In order to achieve high-energy ion acceleration, we must search for the parameters to convert laser energy into ion energy most efficiently. Many studies have been carried out with various gas [5] and solid [6] laser-irradiation targets. In contrast with these studies, we focused on a cluster target [7] because a cluster is an intermediate state between a solid and gas, and we searched for parameters in that domain. It was succeeded with the accelerated energy of $\sim 50$ $\mathrm{MeV} /$ nucleon $\left({ }^{4} \mathrm{He}^{2+}\right)$ using a $\mathrm{CO}_{2}$ cluster in helium background gas [8]. Although this experiment was performed in a radiation-shielded room with 1 meter concrete walls, we had to evaluate the shielding

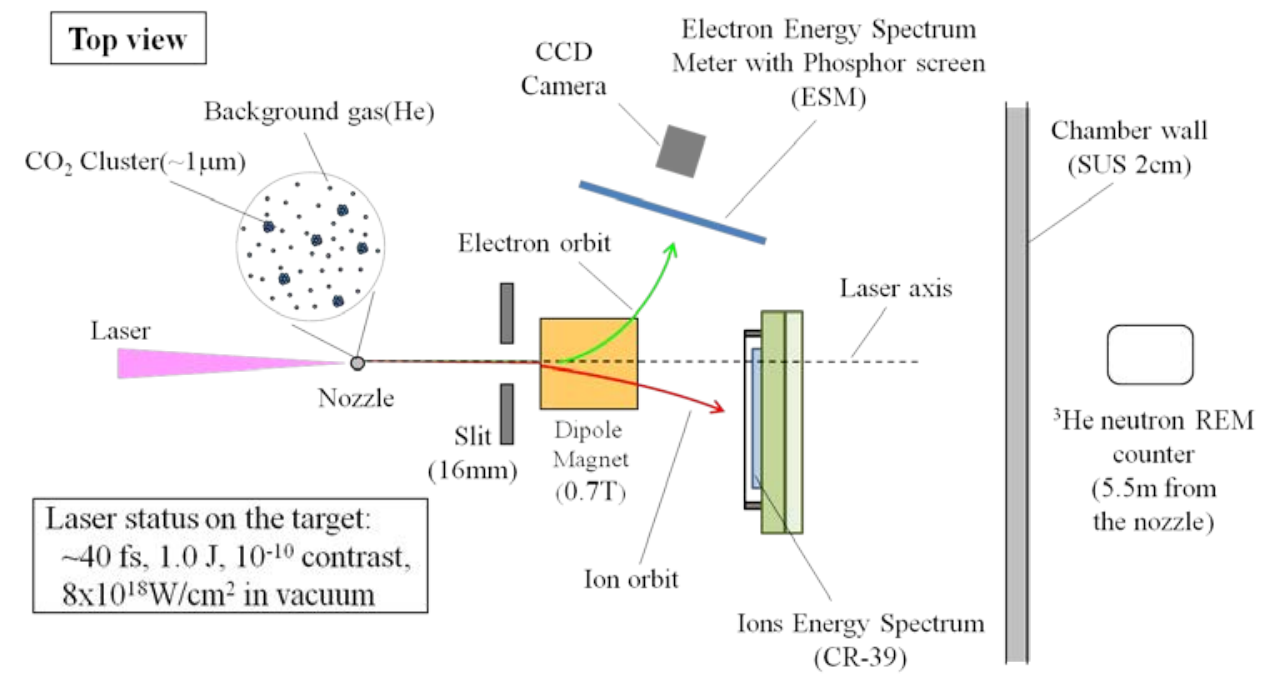

Figure 1. Schematic view of the laser-driven ion acceleration experiment with a cluster target.

\footnotetext{
*Corresponding author. Email: sakaki.hironao@jaea.go.jp
} 
capability of the room from the viewpoint of radiation safety for the accelerated particles. We calculated the dose level using a PHITS input-file to obtain the energy distribution of the particles in the laser-driven acceleration experiment. This result was compared with the doses measured with fluoroglass dosimeters and a helium-3 $\left({ }^{3} \mathrm{He}\right)$ neutron REM counter.

\section{Setup for laser-driven ion acceleration [8]}

Figure 1 shows our experimental setup for laser-driven ion acceleration using $\mathrm{CO}_{2}$ clusters. $\mathrm{CO}_{2}$ clusters are prepared by emitting a mixed gas of $\mathrm{CO}_{2}$ $(10 \%)+\mathrm{He}(90 \%)$ at 60 bar into a vacuum. In this gas condition, the $\mathrm{CO}_{2}$ clusters are intended to grow to $\sim 1$ $\mu \mathrm{m}$. The mixture then is irradiated with a laser at $8 \times 10^{18} \mathrm{~W} / \mathrm{cm}^{2}$ obtained from a J-KAREN system [9]. Online measurement of the electron energy spectrum is carried out with a spectroscope, which uses an electron spectrum meter (ESM) consisting of a 0.7-T magnet, a phosphor screen, and a CCD camera. Moreover, ion energy is measured with a magnet and an offline-type integrating detector (solid-state nuclear track detector: CR-39). We used the ion back-scattering method [10], which can afford a wide measurement range to the CR-39. By using the back-scatter detection technique, we could measure ions with energy exceeding the detection sensitivity of the CR-39. The measurement range for the electron spectrum has a maximum limit of $\sim 200 \mathrm{MeV}$, and ${ }^{4} \mathrm{He}^{2+}$ has the kinetic energy of $\sim 200$ $\mathrm{MeV}$. Neutrons generated from the nuclear reactions are measured with a ${ }^{3} \mathrm{He}$ REM counter (ALOKA TPS-451C) placed $5.5 \mathrm{~m}$ from the nozzle location.

\section{Measurement results}

\subsection{The measurement result for ${ }^{4} \mathrm{He}^{2+}$ ions}

The ${ }^{4} \mathrm{He}^{2+}$ pits on the CR-39, which were measured in the experiment, are shown in Figure 2. When the back-scattering method was used, the ${ }^{4} \mathrm{He}^{2+}$ pits on the CR-39 had an observed energy of $\sim 200 \mathrm{MeV}$ and a number of $\sim 10^{2}$ ions/( $\mathrm{cm}^{2}$ shot). However, the number of ions observed by back-scattering was $\sim 0.01 \%$ of the total [10]. Therefore, it is guessed that the total number of acceleration ions reached about $\sim 10^{5}$ ions $/\left(\mathrm{cm}^{2}\right.$ shot $)$.

\subsection{The measurement result for electrons}

Although calibration is dependent on the sensitivity of the phosphor screen of the ESM, the calibration of this phosphor screen was performed at the maximum energy of the 150-MeV Japan Atomic Energy Agency (JAEA) microtron [11]. The electron energy distribution is shown in Figure 3. These data show a 50 shots average of electric charge with ESM. The electrical charge per pixel is displayed using the accompanying color scale. The maximum energy of electron with a Maxwell distribution was $40 \mathrm{MeV}$. The electron flux

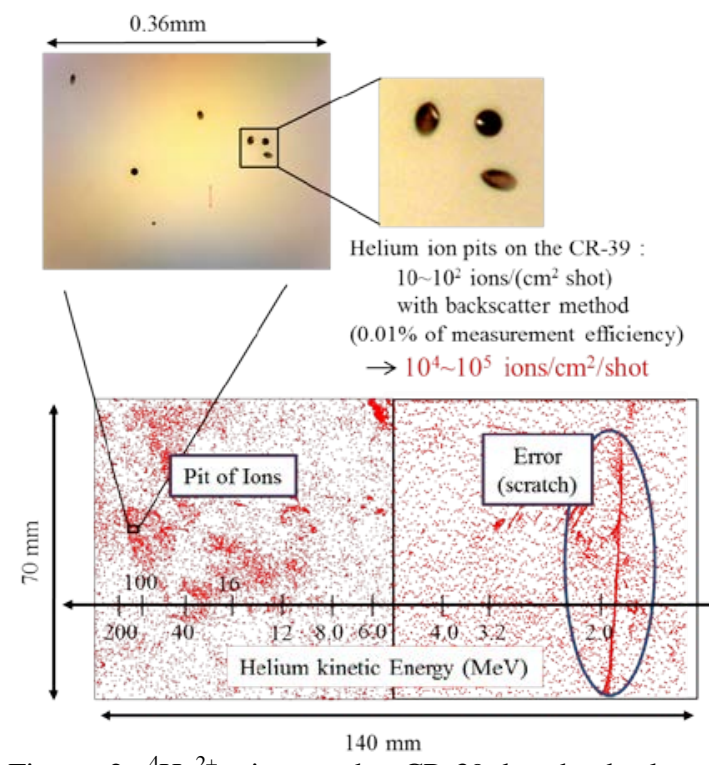

Figure 2. ${ }^{4} \mathrm{He}^{2+}$ pits on the $\mathrm{CR}-39$ by the back-scattering method [8]. Because ions penetrate diagonally due to the influence of back-scatter, most of pits are elliptical.

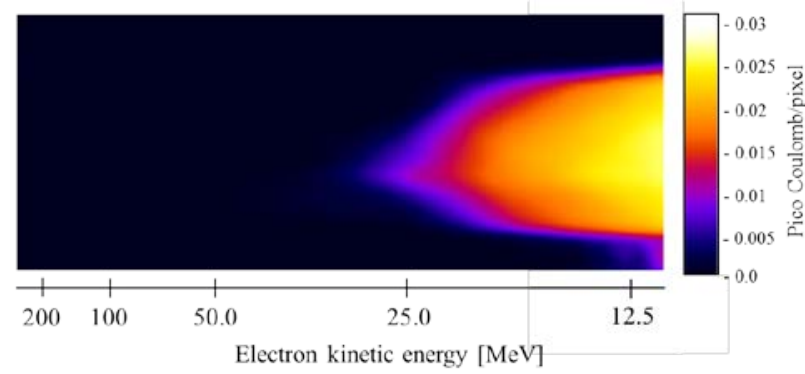

Figure 3. Measurement result for the energy distribution with online ESM.

converted from the electrical charge was $~ 8.0 \times 10^{9}$ electrons $/\left(\mathrm{cm}^{2}\right.$ shot $)$ which is $2-3$ orders greater than the ${ }^{4} \mathrm{He}^{2+}$ flux.

\subsection{The measurement result for the dose}

In order to measure the doses of $x$-rays, $\gamma$-rays, and $\beta$-rays, fluoroglass dosimeters were installed outside of the vacuum chamber at the positions of the CCD and the ${ }^{3} \mathrm{He}$ REM counter as indicated by the red star and triangle in Figure 5. At the star and triangle positions, the dose measurement results of $4.5 \mu \mathrm{Sv} /$ shot and 0.011 $\mu \mathrm{Sv} / \mathrm{shot}$, respectively, were obtained for an average of 12,500 shots. The neutron dose measured by the REM counter $5.5 \mathrm{~m}$ from the nozzle, indicated by a red square in Figure 6, averaged $0.0004 \mu \mathrm{Sv} / \mathrm{shot}$.

\section{Calculated result for radiation dose using PHITS}

\subsection{PHITS}

We analyzed the total of radiation dose from laser-driven acceleration using a general-purpose Monte Carlo PHITS code, [12] which can deal with the transport of electrons, protons, and other ions. Because PHITS 
input includes dipole magnet elements, it also considers details of the beam shown in Figure 1. The photoneutron reaction process in a giant resonance region can be simulated by the PHITS (ver. 2.51 beta) included the Japanese Evaluated Nuclear Data Library Photonuclear Data File 2004 (JENDL/PD-2004). In this calculation, the dose from a single laser shot was analyzed by using electrons and ${ }^{4} \mathrm{He}^{2+}$ as a simulation source.

\subsection{Radiation distribution for PHITS input file}

PHITS requires input the initial particle distribution at the source (nozzle point). Therefore, the energy spectrum, as shown in Figure 4, and angular distribution of the radiation are described in the input file which is obtained from Figures 2 and 3. An electron distribution follows a Maxell distribution, and helium follows a Gaussian distribution. Total source number for this simulation was $3.5 \times 10^{6}$.

\subsection{Calculated result for the radiation dose}

The calculated result expressed by a total dose scale per shot is presented in Figure 5. The calculated result for this part was $\sim 7 \mu \mathrm{Sv} / \mathrm{shot}$ at the location of the red star, and $\sim 7 \times 10^{-3} \mu \mathrm{Sv} / \mathrm{shot}$ at the location of the red triangle. The measured dose at these positions obtained with fluoroglass dosimeters was $4.5 \mu \mathrm{Sv} / \mathrm{shot}$ and 0.011 $\mu \mathrm{Sv} / \mathrm{shot}$, respectively. The neutron dose from both the simulation and the measurement was $<10^{-3} \mu \mathrm{Sv} / \mathrm{shot}$ at the location of red square. So the total-dose causes from $\mathrm{x}$-ray or electron mainly in the simulation was in close agreement with the measurement. Though accelerated particle energy fluctuates according to the shot-shot stability of laser, so one of the simulation errors was caused by laser fluctuation.

The calculated leakage dose at a reference point in

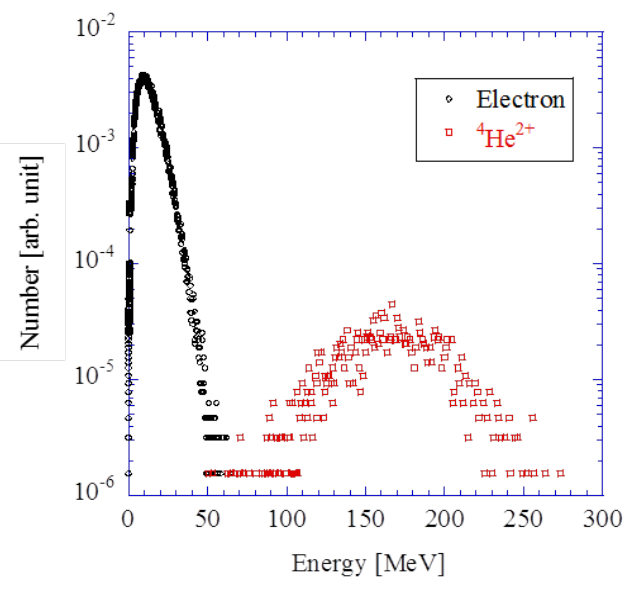

Figure 4. Input source of the energy distribution for the PHITS simulation.

Figure 5 is $\sim 8 \times 10^{-4} \mu \mathrm{Sv} / \mathrm{shot}$ in a rough simulation. Here, the dose-controlled limit outside the shield wall at the reference point indicated by the red circle in Figure 5 is $1.3 \mathrm{mSv} / 3$ months. Since there are $\sim 10^{4}$ shots/day $\left(\sim 10^{3}\right.$ shots/day are in general) in an experiment, the 3 -month total dose at the reference point is $8 \times 10^{-4}$ $\mu \mathrm{Sv} /$ shot $\times 10^{4}$ shots/day $\times 5$ days/week $\times 10$ weeks/3 months $=400 \mu \mathrm{Sv} / 3$ months. The total dose of a background-level, however, has been measured practically by the radiation control manager for every time at the reference point. Accordingly, the shielding is sufficient for this experiment.

\section{Conclusion}

We evaluated the shielding of a laser-driven accelerator by using a PHITS simulation. The simulation

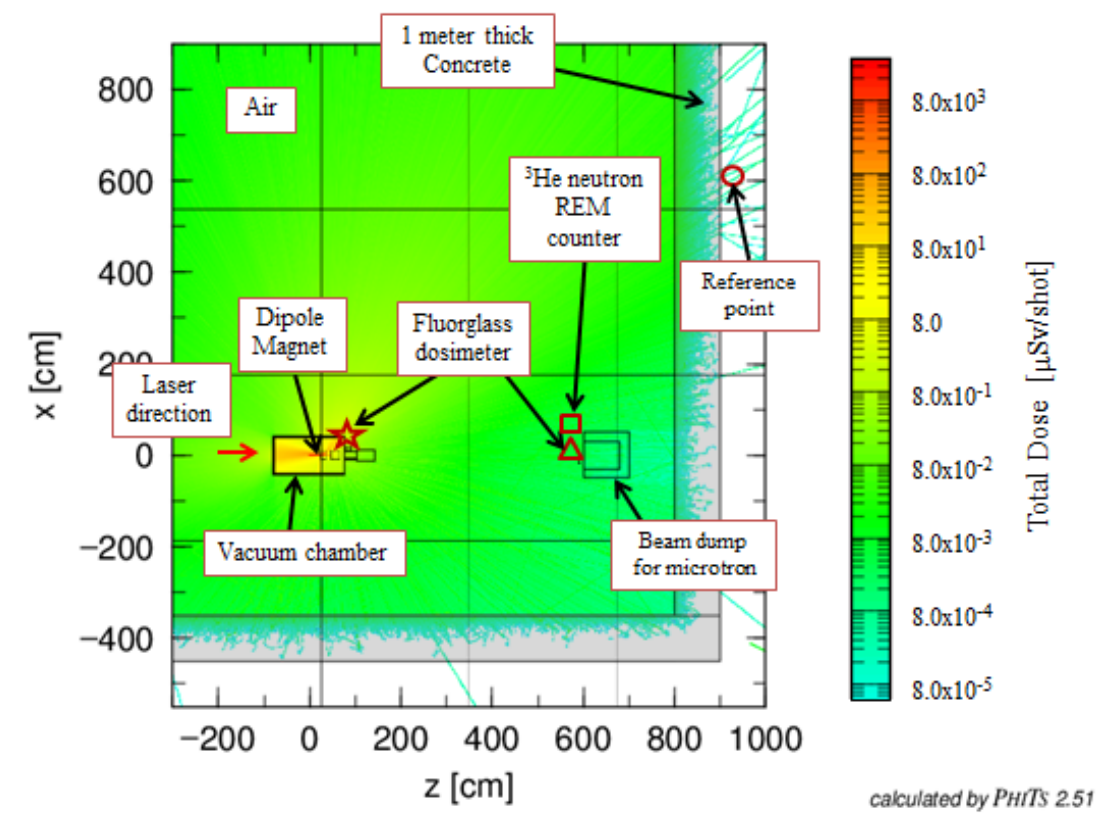

Figure 5. The calculated result for the dose per shot is obtained from the simulation by PHITS. This figure displays the dose distribution result in the $\mathrm{x}-\mathrm{z}$ plane. 
result was in good agreement with the measured doses. In order to increase the accuracy, the radiation process from uncomprehended physics phenomena in the plasma, which was not taken into consideration in this simulation, should be included in a future study.

\section{References}

[1] S.-W. Bahk, P. Rousseau, T. A. Planchon, V. Chvykov, G. Kalintchenko, A. Maksimchuk, G. A. Mourou and V. Yanovsky, Optics Letters 29 (2004), pp.2837-2839.

[2] A. P. Fews, P. A. Norreys, F.N. Beg, A. R. Bell, A. E. Bangor, C. N. Danson, P. Lee and S.J. Rose, Phys. Rev. Lett. 73 (1994), pp.1801-1804.

[3] F. N. Beg, A. R. Bell, A. E. Dangor, C. N. Danson, A. P. Fews, M. E. Glinsky, B. A. Hammel, P. Lee, P. A. Norreys and M. Tatarakis, Phys. Plasmas 4 (1997), pp.447-457.

[4] R. A. Snavely, M. H. Key, S. P. Hatchett, T. E. Cowan, M. Roth, T. W. Phillips, M. A. Stoyer, E. A. Henry, T. C. Sangster, M. S. Singh, S. C. Wilks, A. MacKinnon, A. Offenberger, D. M. Pennington, K. Yasuike, A. B. Langdon, B. F. Lasinski, J. Johnson, M. D. Perry and E. M. Campbell, Phys. Rev. Lett. 85 (2000), pp.2945-2948.

[5] D. Haberberger, S. Tochitsky, F. Fiuza, C. Gong, R. A. Fonseca, L. O. Silva, W. B. Mori and C. Joshi, Nature Physics 8 (2012), pp.95-99.

[6] A. Henig, D. Kiefer, K. Markey, D. C. Gautier, K.
A. Flippo, S. Letzring, R. P. Johnson, T. Shimada, L. Yin, B. J. Albright, K. J. Bowers, J. C. Fernandez, S. G. Rykovanov, H.-C. Wu, M. Zepf, D. Jung, V. Kh. Liechtenstein, J. Schreiber, D. Habs and B. M. Hegelich, Phys. Rev. Lett. 103 (2009), 045002.

[7] Y. Fukuda, A. Ya. Faenov, M. Tampo et al., Phys. Rev. Lett. 103 (2009), 165002.

[8] Y. Fukuda, H. Sakaki, M. Kanasaki, A. Yogo, S. Jinno, M. Tampo, A.Ya. Faenov, T.A. Pikuz, Y. Hayashi, M. Kando, A.S. Pirozhkov, T. Shimomura, H. Kiriyama, S. Kurashima, T. Kamiya, K. Oda, T. Yamauchi, K. Kondo and S.V. Bulanov, Radiati. Measure. 50 (2013), pp.92-96.

[9] H. Kiriyama, M. Mori, Y. Nakai, T. Shimomura, M. Tanoue, A. Akutsu, S. Kondo, S. Kanazawa, H. Okada, T. Motomura, H. Daido, T. Kimura and T. Tajima, Opt. Lett. 33 (2008), pp.645-647.

[10]M. Kanasaki, Y. Fukuda, H. Sakaki, T. Hori, M. Tampo, K. Kondo, S. Kurashima, T. Kamiya, K. Oda and T. Yamauchi, J. J. of App. Phys. 51 (2012), 056401.

[11]I. Daito, M. Kando, H. Kotaki, Y. Hayashi, T. Shizuma, T. Hayakawa, R. Hajima and H. Ohgaki, Proc. IPAC2012 (2012), pp.4124-4126.

[12]K. Niita, N. Matsuda, Y. Iwamoto, H. Iwase, T. Sato, H. Nakashima, Y. Sakamoto and L. Sihver, JAEA-Data/Code 2010-022 (2010). 